\title{
SAMIA : A BOTTOM-UP LEARNING METHOD USING A SIMULATED ANNEALING ALGORITHM
}

\author{
Pierre Brézellec \& Henri Soldano \\ LIPN (CNRS/URA 1507) Université Paris Nord \\ Avenue Jean-Baptiste Clément 93430 Villetaneuse \\ e-mail : brezel@lipn.univ-pariș13.fr \\ $\&$ \\ Institut Curie, section Physique-Chimie, A.B.I \\ 11 , rue Pierre et Marie Curie 75005 Paris.
}

\begin{abstract}
This paper presents a description and an experimental evaluation of SAMIA, a learning system which induces characteristic concept descriptions from positive instances, negative instances and a background knowledge theory. The resulting concept description is expressed as a disjunction of conjunctive terms in a propositional language. SAMIA works in three steps. The first step consists in an exhaustive use of the theory in order to extend the instances representation. Then the learning component combines a bottom-up induction process and a simulated annealing strategy which performs a search through the concept description space. During the final step, the theory is used again in order to reduce each conjunctive term of the resulting formula to a minimal representation. The paper reports the results of several experiments and compares the performance of SAMIA with two other learning methods, namely ID and CN. Accuracies on test instances and concept description sizes are compared. The experiments indicate that SAMIA's classification accuracy is roughly equivalent to the two previous systems. Morever, as the results of the learning algorithms can be expressed as a set of rules, one can notice that the number of rules of SAMIA's concept descriptions is lower than both ID's and CN's one.

Keywords. Bottom-Up Algorithms, Characteristic Descriptions, Concept Learning, Simulated Annealing.
\end{abstract}

\section{Introduction}

During the past few years various systems have been proposed to perform concept learning from examples in the following situation : The goal is to learn concept representations allowing an accurate classification of test sets of objects in two or more classes - Object representation is at propositional level, consisting generally in an attribute-value list - Various kinds of noise can corrupt the data, such as misclassification of examples and random errors on attribute values of examples ; such types of noise are viewed as simple models for difficulties encountered in natural domain learning problems - Concept representations searched for are supposed to be disjunctive ones , i.e., constituted of several conjunctive terms - Both learning set size and object description size can be large.

These systems perform a partial exploration of the concept space to be searched, using various heuristics and some statistical criteria (CN2 [Clark \& Niblett 89], ID3 [Quinlan 86]) or truncation and flexible matching (AQ [Zhang \& Michalski 89]). The concept representations used are either decision trees (ID3), decision lists (CN2) or 
disjunctive formulas (AQ). The search is performed by a top-down generate and test procedure (though AQ uses a data-driven mixed strategy) using specialization operators. For this class of learning problems, little attention has been paid to bottom-up strategies which were considered as inefficient. However, bottom-up techniques have been previously applied to other learning problems concerning noncorrupted data, conjunctive concept representation and first-order object representation ([Mitchell 82], [Kodratoff \& Ganascia 86], [Bisson 92]). In bottom-up methods, the concept representation space is generally restricted to particular conjunctive terms. More precisely, given a concept $\mathrm{C}$ and a set of positive instances of $\mathrm{C}$, a conjunctive term may appear in a formula only if it is obtained by maximal specific generalization of a subset of the positive instances.

The system we propose here is devoted to concept acquisition from positive and negative instances. It describes instances as conjunctions of literals and uses a theory composed of a set of production rules. The theory is used during a preprocessing step in order to extend the description of instances. During the learning step a disjunctive formula is searched for using a simulated annealing optimization procedure. A postprocessing step is then performed in order to obtain minimal representations of the conjunctive terms of the formula. This formula is a characteristic representation of the concept (and not a discriminant one as in top-down methods previously mentioned).

Section 2 presents knowledge representation, properties of the concept representation space, the preprocessing and postprocessing steps and some issues about the learning bias. Section 3 is devoted to the learning component and its simulated annealing algorithm. Section 4 presents experiments on one artificial domain and two natural domains in which SAMIA is compared to $\mathrm{CN}$ and ID. The section 5 reviews related works. The paper ends with a summary and outline of our projects.

\section{Presentation of the System}

Let $A$ be the set of atoms of a given propositional language. $A$ is divided into a set $P$ of primitive atoms and a set $\mathrm{S}$ of non primitive atoms. Moreover a theory $\mathrm{T}$ is given expressed as an unordered set of rules. The left part of each rule is a conjunction of literals of A, and the right part (i.e., the conclusion) is a literal of A. As usual, an instance is defined by the assignation of the values True or False to every atom of A. However each instance is initially described as the conjunction of the corresponding literals of P. The first step of the overall learning mechanism consists of a saturation of every instance with respect to the theory $T$. After the preprocessing step, each instance is represented as the conjunction of the positive literals, where a positive literal is a primitive or a non primitive atom whose value is True. Then, the second step consists in searching for a concept representation $f$ from a set $E_{p}$ of positive instances and a set $E_{n}$ of negative instances. Each element of the search space is a disjunction of conjunctive terms. However only particular conjunctive terms, hereafter denoted as prototypes, are considered. Such terms are maximal specific generalizations of subsets of the set $E_{p}$ of positive instances. In our framework prototypes are recursively defined by an instance or the result of the maximal specific generalization (msg for short) of two prototypes using an intersection operator $\mathrm{msg}$. Let $\mathrm{e}_{1}$ and $\mathrm{e}_{2}$ 
be two prototypes, then $m s g\left(\mathrm{e}_{1}, \mathrm{e}_{2}\right)$ is defined as the conjunction of positive literals 1 belonging to both $e_{1}$ and $e_{2}$. The justification of the restriction of conjunctive terms to maximal specific generalizations relies on the following property :

Proposition 1 : For any term $\mathrm{t}$ (i.e., any conjunction of positive literals of $\mathrm{A}$ ), there exists a prototype $\mathrm{p}$ such that $\mathrm{p}$ covers ${ }^{2}$ exactly the positive instances covered by $\mathrm{t}$ and a subset of the negatives instances covered by $t$.

The prototype $\mathrm{p}$ is simply the maximal specific generalization of the positive instances covered by $t$, and thus is more specific than $t$. This prototype space, together with the general-to-specific partial ordering, defines a "Galois lattice" ( $\left.E_{p}, A\right)$ (Algebraic structure [Ganter, Rindefrey \& Skorsky 86]). Another useful property of the prototypes is related to the logical dependencies between atoms. Such relations may be either explicit ones (i.e., represented inside the theory $\mathrm{T}$ ) or implicit ones.

Proposition 2 : Let $\mathrm{a}_{1}, \ldots, \mathrm{a}_{\mathrm{k}}$ be atoms of $\mathrm{A}$, and assume that the following implication ' $\mathrm{a}_{1} \wedge \ldots \wedge \mathrm{ak}_{\mathrm{k}-1}-->\mathrm{a}_{\mathrm{k}}$ ' holds for a subset $\mathrm{E}$ of the positive instances. Then if a prototype, resulting from the maximal specific generalization of a subset of $\mathrm{E}$, contains $\mathrm{a}_{1}, \ldots, \mathrm{a}_{\mathbf{k}-1}$, then it contains $\mathrm{a}_{\mathbf{k}}$.

Notice that if $\mathrm{E}=\mathrm{E}_{\mathrm{p}}$, then $\mathrm{a}_{\mathrm{k}}$ belongs to any prototype containing $\mathrm{a}_{1}, \ldots, \mathrm{a}_{\mathrm{k}-1}$. As a consequence of the last proposition, we have the following corollary :

Corollary : Let us consider a rule of $\mathrm{T}$ containing only positive literals. If the left part of this rule belongs to a given prototype, then its conclusion also belongs to it.

The property described by the last corollary is the basis of the last step of the learning mechanism. Let $f$ be the formula resulting from the search through the concept representation space. During the postprocessing step, each prototype $p$ of $f$ is rewritten as a conjunctive term with a minimum size and logically equivalent to $p$. This is simply performed by removing from $\mathrm{p}$ every atom which is logically deduced from other atoms of $p$ and rules of the theory. This process has been designed for theories seen as acyclic AND/OR graphs as in [Drastal, Czako \& Raatz 89]. A simple example is used in order to illustrate the whole learning mechanism :

Example :

$\mathrm{P}=\{$ triangle, square, circle, color_black, color_red $\}$,

$\mathrm{S}=\{$ polygon, ellipse $\}$,

$\mathrm{T}=\{$ triangle $->>$ polygon, square $-->$ polygon, circle --> ellipse $\}$

$\mathrm{E}_{\mathrm{p}}=\left\{\mathrm{i}_{1}=\right.$ (triangle, color_red), $\mathrm{i}_{2}=$ (square, color_red), $\mathrm{i}_{3}=$ (square, color_black) $\}$

$\mathrm{E}_{\mathbf{n}}$ is not represented here.

1. Preprocessing step : Both positive and negative instances are extended. $\mathrm{E}_{\mathrm{p}}=\left\{\mathrm{i}_{1}=\right.$ (triangle, polygon, color_red), $\mathrm{i}_{2}=$ (square, polygon, color_red), $\mathrm{i}_{3}=$ (square, polygon, color_black)\}.

2. Searching for a concept representation: Let us suppose that the result $\mathrm{f}$ is as follows : $\mathrm{f}=\mathrm{e}_{1} \vee \mathrm{e}_{2}, \mathrm{e}_{1}=m s g\left(\mathrm{i}_{1}, \mathrm{i}_{2}\right)=($ polygon, color_red $), \mathrm{e}_{2}=m s g\left(\mathrm{i}_{2}, \mathrm{i}_{3}\right)=($ square, polygon).

${ }^{1}$ We will give hereafter a detailed explanation of why negative literals are not allowed in generalizations (It is related to the flexibility of the concept representation).

${ }^{2} \mathrm{~A}$ prototype $\mathrm{p}$ covers an instance $\mathrm{e}$ iff $\mathrm{e} \supseteq \mathrm{p}$ (i.e., $\mathrm{p}$ is more general than $\mathrm{e}$ ). 
3. Postprocessing step : Prototypes are reduced to a minimal form. " $\mathrm{e}_{1}$ " is unchanged, but 'polygon' is removed from e 2 since 'polygon' follows from 'square' and 'square $->$ polygon'. So, we obtain : $\mathrm{f}=$ (polygon, color_red) $\vee$ (square).

Finally, let us remark that when a new instance is processed for a classification purpose, the instance has to be preprocessed in order to be described using the whole language, before it is compared to the prototypes. Another way to check that a prototype p covers an instance $\mathrm{e}$ is to use the initial description of e together with the theory, and to make attempts to prove $\mathrm{p}$.

Negative literals, inductive bias and general attribute-value representation.

In concept learning problems instances are often represented as attribute-value lists such that for each attribute corresponds a value domain D. Various types of attributes are defined, each corresponding to a particular structure of D [Michalsky 84]. Thus for some element of the concept representation space, a given attribute is constrained to a subset of D. The space of allowed constraints on D is usually defined through a generalization operator and represents a form of inductive bias. In our framework each attribute is associated with a set of atoms each corresponding to some subset of D. Then the space of constraints is defined as the space of conjunctions of atomic constraints. As a first example we will consider a non structured value domain D. In this case, when no bias is defined, any subset of $D$ is allowed as a constraint and each value $x$ of $D$ is associated with an atom $X$ representing the subset $D-\{x\}$. Such a representation is a minimal coding of the attribute and has been used in our experiments on natural domain problems with $\mathrm{D}=\{\mathrm{A}, \mathrm{T}, \mathrm{G}, \mathrm{C}\}$ (cf section 4.2 and 4.3). Negative literals are clearly useless here. Moreover some restricted constraint spaces cannot be defined when negative literals are allowed. As an example let us suppose that the user defines a constraint space reduced to the simple subset $\{\mathrm{A}, \mathrm{T}\}$. Then allowing negative literals forces us to add $\{G, C\}$ to the constraints space. A second example is boolean functions learning. Here negative literals are necessary, so they are explicitly represented as new atoms of $\mathrm{A}$. The 11-multiplexer function has been processed this way, using a language containing 22 atoms (cf section 4.1). Considering now structured attributes, the structure may be represented as a part of the theory $T$. This has been shortly exemplified before in the case of a hierarchy. Here again the restriction to positive literals allows more flexibility in defining the constraints space : as an example let us suppose a shape attribute whose value domain $\mathrm{D}$ is structured as follows :

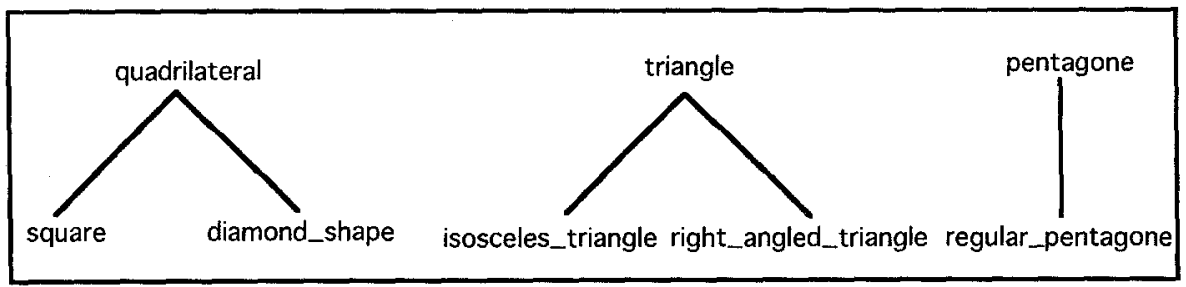

Here the theory is represented as a set of rules. If the negative literals are not allowed, the theory may be restricted to a set of rules ' $x \rightarrow->y^{\prime}$ where $x$ is a node of the hierarchy and $y$ is its parent node. However, if negative literals are allowed, the rules that correspond to the contrapositive (i.e. ' $\neg \mathrm{y} \rightarrow \rightarrow \mathrm{x}$ ' rules) must be added to the theory, together with rules that link roots of the hierarchy as 'quadrilateral --> 
$\neg$ pentagone' and rules expressing constraints as ' $\neg$ square $\wedge \neg$ diamond_shape $-->$ -quadrilateral'.

Now, let us consider the generalization of the pair of instances ((isosceles_triangle), (square)). When no new atoms are added, the generalization leads to the null prototype. This corresponds to the usual bias on hierarchies [Michalski 84]. On the other hand, if all negative literals are represented as new atoms, after preprocessing $m s g$ returns (not_diamond_shape, not_right_angled_triangle, not_regular_pentagone, not_pentagone) which is reduced after the postprocessing step to (not_diamond_shape, not_right_angled_triangle, not_pentagone). A third bias may be considered here by only adding new atoms that correspond to the negation of atoms which are not leaves of the hierarchy. In this case the whole process leads to the prototype (not_pentagone).

Finally defining prototypes as conjunction of positive literals, adding negative literals as new atoms when necessary, and using a theory in order to extend the language, allows a user-defined flexible handling of inductive bias (it has been shown here for the general attribute-value representation case). Furthermore it should be pointed out that the $m s g$ operator clearly has linear computational complexity relatively to the size of the language $\mathrm{A}$. As a consequence the size of the language may be as large as necessary.

\section{The Learning Component}

Simulated Annealing is a method which was proved to be useful in the search of nearoptimal solutions for NP-complete optimization problems [Collins, Eglese \& Golden 88]. This method performs a search for the optimum state of a system according to a given cost function referred to as the energy of the system. A move modifies the current state and then the variation of energy of the system is evaluated. If the move decreases the energy of the system, the new state becomes the current state as in hillclimbing methods ; on the other hand, if the move increases the energy of the system, the new state may become the current state with a probability which depends on the value of a control parameter, denoted as temperature, which decreases slowly during the search.

More precisely, let $\mathrm{E}$ be a finite space of states, $\mathrm{H}$ a cost function of states whose values are positive real numbers, $T$ the temperature, $S$ and $S$ ' states of $E$. Then, our version of the basic sequential simulated annealing algorithm is as follows :

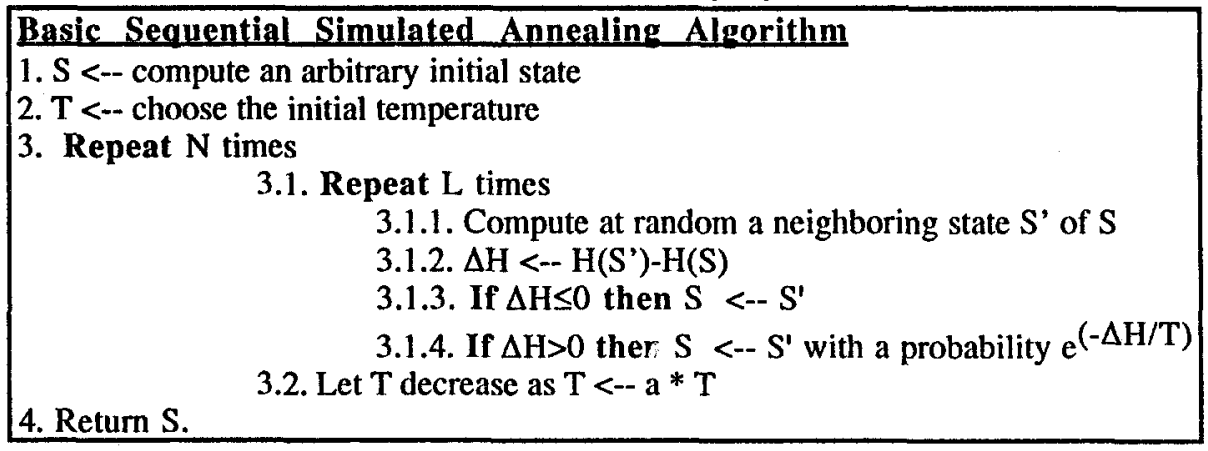

The learning component of SAMIA performs a bottom-up search defined as follows : 
$\mathrm{E}$ is the space of disjunctive formulas whose conjunctive terms are prototypes. $\mathrm{A}$ state $S$ of $E$ is a disjunction of at most NTMAX $^{3}$ terms.

$H(S)$, which represents the cost of a state $S$ of $E$, is defined as the probability of misclassification, and is estimated using examples and counter-examples :

$\mathrm{H}(\mathrm{S})=\mathrm{p}_{\mathrm{ex}}{ }^{* \text { Eerrors }_{\mathrm{ex}}}+\mathrm{p}_{\mathrm{cex}}{ }^{* \text { Eerrors }_{\mathrm{cex}}}$

where,

- $p_{\text {ex }}$ (resp. $p_{c e x}$ ) is the probability of a given object to be a positive instance (resp. negative instance) of the concept,

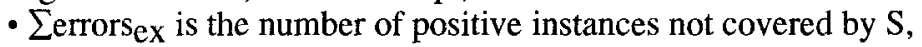

- Eerrors cex is the number of negative instances covered by $S$.

When pex and $p_{c e x}$ are defined as the proportions of positive and negative instances used for learning, we have :

$\mathrm{H}(\mathrm{S})=1-\operatorname{Accuracy}(\mathrm{S})$

where Accuracy is defined as usual (cf section 4).

The initial value of the state $S$ is a disjunctive formula of $\mathrm{NTI}^{4}$ terms. NTI is strictly greater than 1 and lower or equal to NTMAX. The terms of the initial state are randomly selected positive instances.

A neighbor $S^{\prime}$ of a state $S$ results from the application of a randomly selected operator to $S$. Let $\mathrm{n}$ be the number of terms of $\mathrm{S}$. Then the operators used here are defined as follows :

$1^{\circ}$ ) Extension operator: If $\mathrm{n}$ is strictly lower than NTMAX, the extension operator appends a new term to $S$; this term is a randomly selected positive instance,

$\left.2^{\circ}\right)$ Contraction operator: The contraction operator removes a randomly selected term from $\mathrm{S}$,

$\left.3^{\circ}\right)$ Generalization operator: The generalization operator randomly selects a term $t$ of $S$ and performs the maximal specific generalization of $t$ with a randomly selected positive instance $\mathrm{e}$. If the resulting term $m s g(t, e)$ covers a ratio of negative instances smaller than a consistency threshold value $\mathrm{PCEX}^{5}$, then a new state is generated by replacing the term $t$ by $m s g(t, e)$. Checking consistency of the new term is useful since no specialization operator is available.

Finally, before returning the result S, SAMIA discards each term more specific than at least one other term. This step eliminates redundancies.

Before describing the experiments, we give below an example of a curve which shows the behavior of SAMIA (i.e., its accuracy on the learning set) with respect to $\mathrm{N}$ (i.e., the number of iterations, here set up to 2600) during the learning step of the concept "11-multiplexer" :

\footnotetext{
${ }^{3}$ NTMAX is a user parameter.

${ }^{4}$ NTI is a user parameter.

${ }^{5} \mathrm{PCEX}$ is a user parameter.
} 


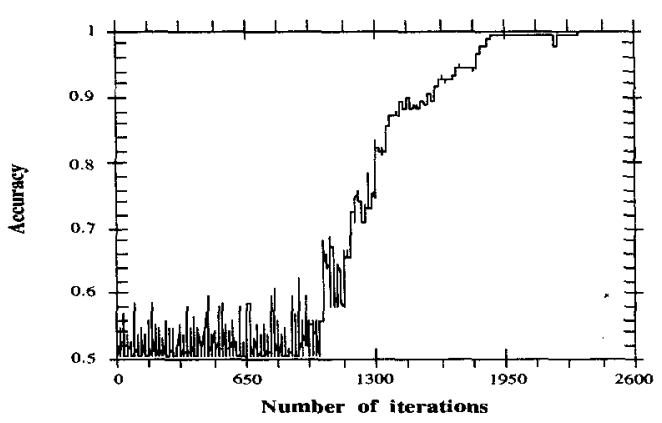

\section{Experiments}

The learning component of SAMIA together with ID and $\mathrm{CN}^{6}$ have been tested on one artificial domain problem, the 11-multiplexer problem, and two natural domain problems issued from DNA sequence analysis (learning of promoters and 5'-splice sites). This twofold evaluation is motivated by considering that 1) Artificial domains let us control the environment of the experiments. Thus, it is easy to introduce varying degrees of corruption of attributes values or of class membership values, 2) Generally, in natural domain such as experimental data classification or pattern recognition problems, neither the relevant object description nor the proper form of an accurate concept description are known.

Two aspects of the algorithms' behavior have been used to assess them :

$\left.1^{\circ}\right)$ Classification accuracy : Given a set of instances where true classification is known, the accuracy of a concept description is the estimated percentage of correct classification (i.e., ACC is the number of correct classifications divided by the number of instances tested).

$\left.2^{\circ}\right)$ Syntactic complexity of the concept representations [Zhang \& Michalski 89] : SAMIA, ID and $\mathrm{CN}$ have different concept description languages. These are the Disjunctive Formulas, Decision Trees, and Decision Lists. The syntactic complexity of the descriptions are computed as follows :

-The syntactic complexity of a disjunctive formula is NT*ANS where NT is the number of terms of the disjunctive formula and ANS is the average number of literals in the conjunctive terms,

-The syntactic complexity of a decision tree is $\mathrm{NN}$ where $\mathrm{NN}$ is the number of its nodes (including leaves); NL is the number of the leaves of the decision tree,

-The syntactic complexity of a decision list is NR*ANS where NR is the number of rules of the decision list and ANS is the average number of selectors in the rules.

We enumerate below ID's (with and without pruning), CN's and SAMIA's uservariable parameters which are used in our experiments.

-ID without pruning (see [Quinlan 86]) : Prune threshold $=0 \%$, Variance termination threshold $=10 \%$,

-ID with pruning : Prune threshold $=10 \%$, Variance termination threshold $=10 \%$,

${ }^{6}$ ID and CN [Boswell 90ab] are respectively Boswell's implementations of the ID3 [Quinlan 86] and CN2 [Clark \& Niblett 89] algorithms. 
-CN (see [Clark \& Niblett 89]): Threshold of significance $=1$, Star size $=5$, -SAMIA : $\mathrm{N}=2600, \mathrm{~L}=8, \mathrm{a}=0.996, \mathrm{NTMAX}=20, \mathrm{NTI}=2$, PCEX $=0.02$, except in the "5'-splice" sites problem where it is set up to 0.0 .

\subsection{The 11-multiplexer Problem}

The 11-multiplexer boolean function has often been used to evaluate learning methods ([Wilson 87], [Quinlan 88], [Van de Velde 89], [Zhang \& Michalski 89]). Each object is a boolean vector divided into 3 address bits and $2^{3}$ data bits. An object is a positive instance of the 11-multiplexer function if the data bit whose index in the vector corresponds to the address bits has value 1 . The 11-multiplexer function can be represented as a normal disjunctive form ( 8 terms of 4 literals each (i.e., $\left(x_{1}=0 \wedge\right.$ $\left.\left.\left.\mathrm{x}_{2}=0 \wedge \mathrm{x}_{3}=0 \wedge \mathrm{x}_{4}=1\right) \vee \ldots \vee\left(\mathrm{x}_{1}=1 \wedge \mathrm{x}_{2}=1 \wedge \mathrm{x}_{3}=1 \wedge \mathrm{x}_{11}=1\right)\right)\right)$.

In order to evaluate SAMIA, ID and $\mathrm{CN}$ in noisy environments, two kinds of noise have been introduced:

$\left.1^{\circ}\right)$ Noise of level $n$ on class membership values : $n$ percent of the positive instances and $n$ percent of the negative instances are randomly selected; then, the value of the class of each selected instance is modified.

$2^{\circ}$ ) Noise of level $n$ on attribute values : n percent of the positive instances and $n$ percent of the negative instances are randomly selected ; then, the value of a randomly chosen attribute of each selected instance is modified.

The instances of the test sets are not corrupted ([Clark \& Niblett 89], [Rendell \& Cho 90]).

The experimental design is defined as follows:

Step-1 : Random selection of 200 positive learning instances and 200 negative learning instances of the 11-multiplexer function.

Step-2: Corruption of the learning sets according to the kind and level of noise.

Step-3 : Learning using SAMIA, ID (with and without pruning) and CN.

Step-4: Classification of test instances (the 1648 positive and negative instances left out in step 1).

This procedure is repeated 40 times.

Table 1 presents the results of each algorithm for varying degrees of corruption of class membership values (the attribute value corruption level is 0 ). In each case, we present the average accuracy on the test data and the average syntactic complexity of the resulting concept descriptions.

table 1

\begin{tabular}{|c|c|c|c|c|c|c|c|c|c|c|c|c|}
\hline \multirow{2}{*}{$\begin{array}{l}\text { class } \\
\text { error }\end{array}$} & \multicolumn{3}{|c|}{$\begin{array}{l}\text { SAMIA } \\
\text { PCEX } 0.02 \\
\end{array}$} & ID with & \multicolumn{3}{c|}{$\begin{array}{l}\text { ID without } \\
\text { pruning }\end{array}$} & \multicolumn{3}{c|}{ CN } \\
\cline { 2 - 14 }$y$ & ACC & NT & ANS & ACC & NL & NN & ACC & NL & NN & ACC & NR & ANS \\
\hline 0 & 0.959 & 11.5 & 4.3 & 0.921 & 83.8 & 166.7 & 0.904 & 78.3 & 155.7 & 0.986 & 18.7 & 2.8 \\
\hline 5 & 0.907 & 16.9 & 4.9 & 0.841 & 119.6 & 238.3 & 0.84 & 104 & 207.4 & 0.873 & 41.4 & 3.4 \\
\hline 10 & 0.809 & 19.3 & 4.9 & 0.762 & 145.5 & 290 & 0.77 & 132.6 & 264.3 & 0.79 & 51.8 & 3.6 \\
\hline 15 & 0.765 & 19.4 & 5 & 0.725 & 167.3 & 333.6 & 0.733 & 154.7 & 308.5 & 0.742 & 59.2 & 3.8 \\
\hline 20 & 0.702 & 19.8 & 5 & 0.678 & 183.7 & 366.4 & 0.685 & 174.4 & 347.9 & 0.695 & 65.5 & 3.9 \\
\hline
\end{tabular}


Table 2 presents the results of each algorithm for varying degrees of corruption of attribute values (class membership corruption level is 0 ).

table 2

\begin{tabular}{|c|c|c|c|c|c|c|c|c|c|c|c|c|}
\hline \multirow{2}{*}{$\begin{array}{l}\text { attribute } \\
\text { error }\end{array}$} & \multicolumn{3}{|c|}{$\begin{array}{c}\text { SAMIA } \\
\text { PCEX } 0.02 \\
\end{array}$} & \multicolumn{3}{|c|}{ ID without } & \multicolumn{3}{c|}{$\begin{array}{c}\text { ID with } \\
\text { pruning }\end{array}$} & \multicolumn{3}{c|}{ CN } \\
\cline { 2 - 15 } & ACC & NT & ANS & ACC & NL & NN & ACC & NL & NN & ACC & NR & ANS \\
\hline 5 & 0.95 & 12.9 & 4.6 & 0.883 & 97.5 & 194 & 0.87 & 85.8 & 170.6 & 0.959 & 25.2 & 2.8 \\
\hline 10 & 0.939 & 13.9 & 4.6 & 0.866 & 108.4 & 215.9 & 0.86 & 93 & 185 & 0.933 & 31.3 & 2.9 \\
\hline 15 & 0.917 & 15.7 & 4.8 & 0.869 & 103.8 & 206.7 & 0.873 & 81.9 & 163 & 0.898 & 37.7 & 3.2 \\
\hline 20 & 0.929 & 15.8 & 5 & 0.838 & 112.7 & 224.4 & 0.841 & 85.4 & 170.3 & 0.883 & 39.3 & 3.4 \\
\hline
\end{tabular}

\subsection{The "Promoter" Problem}

The genome of the Prokaryotes, i.e., bacteria for instance, can be considered as a string which is composed of coding regions -i.e., genes- and non coding regions. A promoter is a particular region which initiates the first step in the expression of an adjacent gene. The set of positive and negative instances of promoters of the Escherichia Coli bacteria that we use in our experiment is described in [Towell, Shavlik \& Noordewier 90]. It contains 53 positive instances and 53 negative instances. Each instance is composed of 57 attribute-value pairs whose value domains are $\{\mathrm{A}, \mathrm{T}, \mathrm{G}, \mathrm{C}\}$. A standard leave-one-out method has been used in this experiment. Let $\mathrm{N}$ be the total number of instances, then the training is performed using $\mathrm{N}-1$ instances, and the remaining instance is classified. This procedure is repeated $\mathrm{N}$ times, so that each instance is excluded once from the training set. The whole procedure was repeated 10 times for SAMIA whose results are non-deterministic, and an average accuracy was computed.

Table 3 summarizes the experimental results.

table 3

\begin{tabular}{|c|c|c|c|c|c|c|c|c|c|c|c|c|}
\hline \multirow{3}{*}{$\begin{array}{l}\text { promoter } \\
\text { problem }\end{array}$} & \multicolumn{3}{|c|}{$\begin{array}{l}\text { SAMIA } \\
\text { PCEX } 0.02\end{array}$} & \multicolumn{3}{|c|}{$\begin{array}{l}\text { ID without } \\
\text { pruning }\end{array}$} & \multicolumn{3}{|c|}{$\begin{array}{l}\text { ID with } \\
\text { pruning }\end{array}$} & \multicolumn{3}{|c|}{$\mathrm{CN}$} \\
\hline & $\mathrm{ACC}$ & NT & ANS & $A C C$ & NL & NN & $A C C$ & NL & NN & $\mathrm{ACC}$ & NR & ANS \\
\hline & 0.814 & 3.6 & 10.92 & 0.773 & 30.9 & 40.8 & 0.792 & 24.8 & 32.7 & 0.83 & 9.1 & 1.2 \\
\hline
\end{tabular}

In this problem, as in the next one, in order to allow fair comparison of syntactic complexities, SAMIA's prototypes are translated from the propositional language to constraints on the value domain of the attributes. Then a prototype is a conjunction of constraints $\left(a_{i} \in S_{i}\right)$, where $S_{i}$ denotes a subset of $\{A, T, G, C\}$, and $a_{i}$ the $i$ th attribute. Comparison with ID is still unfair since each node is associated with four possible outcomes, namely $\{\mathrm{A}\},\{\mathrm{T}\},\{\mathrm{G}\},\{\mathrm{C}\}$.

\subsection{The "5'-splice site" Problem}

The genes of the Eukaryotes (i.e humans and leeks for instance) are split. Thus, these genes are composed of coding regions -called exons- and non coding regions -called introns-. A "5'-splice site" is a region that directs the excision of the introns [Brunak, 
Engelbrecht \& Knudsen 91]. The training set used here contains 302 positive instances and 600 negative instances of 5'-splice sites. A test set containing 118 positive instances and 600 negative instances is also provided. Each instance is described using 18 attribute-value pairs whose value domains are $\{A, T, G, C\}$. The procedure is repeated 40 times for SAMIA.

Table 4 summarizes the experimental results.

\section{table 4}

\begin{tabular}{|c|c|c|c|c|c|c|c|c|c|c|c|c|}
\hline \multirow{3}{*}{$\begin{array}{l}5 \text { '-splice } \\
\text { site } \\
\text { problem }\end{array}$} & \multicolumn{3}{|c|}{$\begin{array}{l}\text { SAMIA } \\
\text { PCEX } 0.0\end{array}$} & \multicolumn{3}{|c|}{$\begin{array}{l}\text { ID without } \\
\text { pruning }\end{array}$} & \multicolumn{3}{|c|}{$\begin{array}{l}\text { ID with } \\
\text { pruning }\end{array}$} & \multicolumn{3}{|c|}{$\mathrm{CN}$} \\
\hline & $\mathrm{ACC}$ & NT & ANS & $\mathrm{ACC}$ & NL & NN & $\mathrm{ACC}$ & NL & NN & $A C C$ & NR & ANS \\
\hline & 0.88 & 19.8 & 12.4 & 0.84 & 214 & 285 & 0.89 & 89 & 121 & 0.88 & 64 & 2.3 \\
\hline
\end{tabular}

\subsection{Discussion}

The first comment is that, roughly speaking, the accuracies of the three systems are equivalent. This is interesting since the three problems are very different. The concept representation space of the 11 -multiplexer is rather small and the concept is highly disjunctive. Many useless attributes are used to describe the promoter problem and few instances are provided. "5'-splice sites" are described with a relatively small number of attributes and many instances are provided. Comparing the sizes of the concept representation learned by the three systems, it should be noticed that SAMIA produces less rules; in the case of the noise free 11-multiplexer problem SAMIA is closed to the optimal solution regarding both the number of rules and the average rule length. Moreover, it should be pointed out that SAMIA seems to slightly better handle misclassification noise. Concerning the two other experiments, full comparison of syntactic complexities is difficult. However, the average number of components in SAMIA's rules is larger than CN's one. This is not surprising since SAMIA searches for characteristic concept descriptions.

Finally, the table bellow reports the average elapsed cpu time (seconds), during learning 7 :

\begin{tabular}{|c|c|c|c|}
\hline cpu time & 11 -multi. & Promoter & 5'-splices \\
\hline ID & 1.92 & 2 & 4 \\
\hline CN & 20 & 90 & 101 \\
\hline SAMIA & 350 & 433 & 1922 \\
\hline
\end{tabular}

The run times reveal that SAMIA is the slowest of the three algorithms. This is mainly due to the simulated annealing search strategy that requires more computation time to solve complex problems. However, a large research effort focuses on parallel implementations of this strategy [Roussel-Ragot 90].

${ }^{7}$ SAMIA, ID, and CN were run on a SUN/Sparc 1. 


\section{Related Works}

Concerning the concept representation space, our approach is close to the work of [Kudo \& Shimbo 89]. In this work the authors search for all maximal subsets of the positive instances whose maximal specific generalizations cover no negative instances. Their algorithm seems efficient but still has exponential complexity with the number of positive instances since it performs an exhaustive search. In another related work, learning is performed by constructing a Gallois lattice [Liquière \& Méphu Nguifo 90]. The construction is only partial thanks to the use of a consistency criterion. As a matter of fact the nodes of the lattice represent prototypes as defined here. Unfortunately, here again exhaustive search leads to intractable computational complexity. From this point of view the simulated annealing strategy seems to limite the following shortcoming of bottom-up methods : although the concept representation space is restricted, the space which is practically searched may be much larger, since search is performed in a space whose elements are subsets of instances. Finally, one can note that the redundancies of the description language, which are unavoidable when using background knowledge in order to perform an exhaustive deductive step, have only limited effect in such methods, since the computational complexity of the generalization operator is linear with the size of the language.

Concerning the use of background knowledge, and the control of learning bias, full evaluation of our method has not yet been performed. In the future, we intend to compare our system to MIRO [Drastal, Czako \& Raatz 89] in which the theory is used to move the description language toward a more abstract one, rather than extending the language as it is done here.

\section{Conclusion}

The most significant aspects of our method is that 1) it combines a bottom-up approach and simulated annealing search strategy, 2) it uses background knowledge in a preprocessing level in order to extend instance description as do various other learning systems ([Rouveirol 90], [Brézellec \& Champesme 91], [Bisson 92]), 3) it uses background knowledge in a postprocessing level in order to obtain a minimal representation of the conjunctive terms of the concept description.

The experiments we have conducted show that ID, CN and SAMIA have comparable performance. Moreover, since SAMIA'smsg operator has linear computational complexity relatively to the size of the language, we think that it will be well suited to situations in which an exhaustive deductive step is applied to the learning instances.

At present, we plan to add other operators to SAMIA. For instance, we think that an operator that will undo a generalization step is necessary to improve SAMIA's efficiency. Indeed, such an operator will decrease the "gap" between a state and its neighborhood, and therefore the search space will be "smoother".

Finally, we intend to examine other optimization algorithms ("Taboo search" for instance) and their relations to learning. As in this work, it will require some ideas to fit a learning problem to an optimization one.

\section{Acknowledgments}

We thank the LRI of Orsay (France) for giving us ID and CN, and the "UCI Repository Of Machine Learning Databases and Domain Theories" for supplying the 
"promoter" data set. Special thanks go to Alain Viari (Institut Curie/CNRS) for his invaluable assistance in understanding the two biological problems and to Alex Simionovici for correcting various syntactical english errors of our paper. This work has been supported by a grant from ORGANIBIO ( $\mathrm{Cm} 2 \mathrm{aO}$ program).

\section{References}

[Bisson 92] Gilles Bisson, "Conceptual Clustering in a First Order Logic Representation", Tenth European Conference on Artificial Intelligence, Vienna 92, Bernd Neumann, pp 458-468.

[Boswell 90a] J. Boswell, "Manual for NewID version 2.1", The Turing Institute, January 1990.

[Boswell 90b] J. Boswell, "Manual for CN2", The Turing Institute, January 1990.

[Brunak, Engelbrecht \& Knudsen 91] Soren Brunak, Jacob Engelbrecht, Steen Knudsen, "Prediction of Human mRNA Donor and Acceptor Sites from the DNA Sequence", Journal of Molecular Biology (n²20, 1991), pp 49-65.

[Brézellec \& Champesme 92] Pierre Brézellec, Marc Champesme, "Vers un système d'apprentissage moins sensible au bruit, et aux descriptions et théories initiales", Huitième Congrès Reconnaissance des Formes et Intelligence Artificielle, LyonVilleurbanne 91, pp 945-952.

[Collins, Eglese \& Golden 88] N.E. Collins, R.W. Eglese, B.L. Golden, "Simulated annealing - An annotated bibliography", American journal of mathematical and management sciences 8, pp 209-307.

[Clark \& Niblett 89] Peter Clark, Tim Niblett, "The CN2 Induction Algorithm", Machine Learning (volume 3, number 4, March 89), Kluwer Academic Publishers, pp 261-283.

[Drastal, Czako \& Raatz 89] G. Drastal, R. Czako, S. Raatz, "Induction in an abstraction space : A form of constructive induction", Proceedings of the Eleventh International Joint Conference on Artificial Intelligence, Detroit 89, Morgan Kaufmann, pp 708-712.

[4] Ganter B., Rindefrey K., Skorsky M. "Software for a formal concept analysis", Classification as a tool of Research, North Holland 1986.

[Liquière \& Méphu Nguifo 90] Michel Liquière, Engelbert Méphu Nguifo, 'LEarning with GAlois Lattice : Un système d'apprentissage de concepts à partir d'exemples", Cinquièmes Journées Françaises d'Apprentissage, Lanion 90, pp 93-113.

[Kodratoff \& Ganascia 86] Yves Kodratoff, Jean-Gabriel Ganascia, "Improving the generalization step in Learning", Machine Learning, An Artificial Approach (volume II), Morgan Kaufman (1986), pp 215-244.

[Kudo \& Shimbo 89] Mineichi Kudo, Masaru Shimbo, "Optimal subclasses with dichotomous variables for features selection and discirmination", IEEE Trans. Systems, Man, Cybern., 19, pp 1194-1199.

[Michalski 84] R.S. Michalski, "A Theory and Methodology of Inductive Learning", Machine Learning : An Artificial Approach (volume I), Springer Verlag (1984), pp 83-129.

[Michalski \& al 86] Ryszard S. Michalski, Igor Mozetic, Jiarong Hong, Nada Lavrac, "The multi-purpose incremental learning system AQ15 and its testing application to three medical domains", Proceedings of the Fifth National Conference on Artificial Intelligence, Morgan Kaufman, pp 1041-1045. 
[Quinlan 86] J.R. Quinlan, "Induction of Decision Trees", Machine Learning (volume 1, number 1, 1986), Kluwer Academic Publishers, pp 81-106.

[Quinlan 88] J.R. Quinlan, "An Empirical Comparison of Genetic and Decision-Tree Classifier", Proceedings of the Fifth International Conference on Machine Learning, Ann Arbor 88, pp 135-141.

[Rendell \& Cho 90] Larry Rendell, Howard Cho, "Empirical Learning as a Function of Concept Character", Machine Learning (volume 5, number 3, August 90), Kluwer Academic Publishers, pp 267-298.

[Roussel-Ragot 90] P. Rousel-Ragot, "La méthode du recuit simulé : Accélération et parallélisation", Thèse de doctorat de l'Université Paris 6.

[Rouveirol 90] Celine Rouveirol, "Saturation : Postponing Choices when Inverting Resolution", Seventh International Conference on Machine Learning, Austin 90, pp 557-562.

[Towell, Shavlik \& Noordewier 90] Geoffrey G. Towell, Jude W. Shavlik, Michiel O. Noordewier, "Refinement of Approximate Domains Theories by Knowledge-Based Neural Networks", AAAI 90, pp 861-866.

[Van de Velde 89] Walter Van de Velde, "IDL, or Taming the Multiplexer", Proceedings of the Fourth European Working Session on Learning, Montpellier 89, Morgan Kaufmann, pp 211-225.

[Wilson 87] S. W. Wilson, "Classifier Systems and the Animat Problem", Machine Learning (volume 2, number 4, 1987), Kluwer Academic Publishers, pp 199-226.

[Zhang \& Michalski 89] Jianping Zhang, Rysard S. Michalski, "Rule Optimization Via SG-Trunc Method", Proceedings of the Fourth European Working Session on Learning, Montpellier 89, Morgan Kaufmann, pp 251-262. 